\title{
LA JUSTICIABILIDAD DE LOS DERECHOS HUMANOS. UNA LECTURA EN CLAVE DE LOS PROBLEMAS CLÁSICOS DE LA POLÍTICA SOCIAL
}

\author{
Pilar Arcidiácono \\ Universidad de Buenos Aires / CONICET \\ Gustavo Gamallo \\ Universidad de Buenos Aires
}

http://dx.doi.org/10.5209/NOMA.53307

\begin{abstract}
Resumen: En los últimos años en América Latina se advirtió el incremento de la participación del poder judicial como ámbito para dirimir la satisfacción de demandas vinculadas con derechos sociales en un contexto más general de protagonismo de los derechos en la escena pública. Los trabajos que frecuentemente abordaron estas temáticas tendieron a soslayar el lugar de la política pública (especificamente social) y de la economía de mercado. La hipótesis que guía el artículo es que la forma compleja y cambiante de la relación entre las aspiraciones igualitarias de los derechos sociales y los mecanismos de provisión de bienes y servicios en sociedades de mercado pueden comprenderse mejor a partir de una mirada más próxima a la política social y la economía política del bienestar. Este aporte permite introducir los conceptos de mercantilización y desmercantilización como dimensiones centrales de análisis para comprender los procesos políticos institucionales que dan forma a la provisión de satisfactores de necesidades sociales y están mediados conflictivamente por la lógica de los derechos.
\end{abstract}

Palabras clave: Derechos sociales-justiciabilidad-politicas sociales-satisfactoresdesmercantilización.

\section{The Role of Social Rights and Justiciability. A Reading From the Classic Key Issues of Social Policy}

Abstract: In recent years in Latin America the role of rights and increased participation of the judicial branch as the satisfaction level to settle claims related to social rights it was increased. The academic papers often addressed these issues tended to overlook the place of public policy (specifically social policy) and market economy. The hypothesis that guides this article is that the complex and changing relationship between the egalitarian aspirations of social rights and the mechanisms for providing goods and services in capitalist societies-or in other words the scope and limitations of the rights and the actions of the justice- may best be understood from a closer to the social policy and welfare economics approach. This contribution allows to introduce the concepts of commodification and decommodification as central dimensions of analysis to understand the institutional and political processes that shape the provision of satisfaction of social needs and are mediated by the conflictive logic of rights.

Keywords: Social rigths-justiciability-social policies-satisfactors-de-commodification 


\section{Introducción}

En los últimos años se advirtió en la regiónel incremento de la participación del poder judicial como ámbito para dirimir la satisfacción de demandas vinculadas con derechos sociales. Surgieron sentencias relevantes sobre el derechos a la educación, a la salud,a la vivienda y a la seguridad social por parte de tribunales argentinos, costarricenses, brasileños y colombianos, por nombrar algunos (Arcidiáconoet al, 2010) ${ }^{1}$.

Dicho proceso fue facilitado, por un lado, por reformas constitucionales que recogieron los instrumentos internacionales de derechos humanos, y por otro lado y en un sentido negativo, por debilidades, incapacidades y resistencias de los poderes legislativos y ejecutivos para atender reclamos insatisfechos. En ese contexto el "discurso de los derechos" fue adquiriendo protagonismo en las peticiones ciudadanas a la vez que el llamado "enfoque de los derechos humanos" influyó en el diseño y evaluación de las políticas sociales (Abramovich y Pautassi, 2009; Gargarella, 2006; AUTOR, 2008).

Los estudiossobre la temática sostienenmayoritariamente enfoques jurídicos. ${ }^{2}$ Frecuentemente suelen restarle atención al lugar de la política estatal (específicamente social) y de la economía de mercado minimizando tanto los conflictos que atraviesa el diseño y la implementación de las políticas comolos problemas asociados a la provisión pública de bienes y servicios sociales en contextos de restricción fiscal y competencia por recursos escasos.

El artículo se propone una reflexión conceptual sobre la forma compleja y cambiante de la relación entre las aspiraciones igualitarias de los derechos sociales y los mecanismos de provisión de bienes y servicios en sociedades de mercado. Nuestro aporte, próximo a los estudios dela política social y la economía política del bienestar,introduce los conceptos de mercantilización y desmercantilización(Esping Andersen, 2000) como dimensiones analíticas centrales para comprender los procesos políticos institucionales que dan forma a la provisión de

\footnotetext{
${ }^{1}$ A nivel mundial, se encuentra la jurisprudencia de la Corte Suprema de la India, que abordó temas sociales estructurales como el hambre y el analfabetismo, y que se ha acompañado de la creación de comisiones judiciales de consulta para hacerle seguimiento a la implementación de los fallos. También la Corte Constitucional sudafricana se ha convertido en un espacio institucional para la promoción de derechos como la vivienda y la salud, y también de presión para que el estado actúe en contra del legado económico y social del apartheid.

${ }^{2}$ En Argentina algunos trabajos comenzaron a indagar sobre la problemática de la judicialización de la política (no necesariamente la política social) desde el campo de la teoría política intentando con éxito desmarcarse del "saber jurídico" (Martin, 2012; Nosetto, 2014).
} 
satisfactores de necesidades sociales,mediados conflictivamente por la lógica de los derechos. ${ }^{3}$

\section{Una breve advertencia}

Es sabido, y la siguiente advertencia tal vez sea redundante e innecesaria, que la expansión del derecho racional formal fue un proceso paralelo y constitutivo del capitalismo contemporáneo y de la conformación de los estados nacionales (Weber, 1944). El derecho estableció mecanismos de reciprocidad entre gobernantes y gobernados en sociedad que ya no podían fundar la dominación solamente en base a la coacción (Giddens, 2006). O`Donnell (2008a:28) defendió que el Estado moderno es también un "sistema legal, un entramado de reglas que penetran y co-determinan numerosas relaciones sociales". Las características postuladas por ese sistema legal son la universalidad y la generalidad y constituyen la base necesaria para la construcción de las expectativas recíprocas en los intercambios sociales. Escrito en 1900, en uno de sus pocos textos sobre el problema del Estado, Émile Durkheim señalaba: "En todo momento se ve engrosarse progresivamente el volumen de los Códigos, lo que prueba que el derecho penetra en las esferas de la vida social en las que anteriormente se encontraba ausente, y lo hace cada vez con mayor profundidad, sometiendo a su acción toda clase de relaciones que le estaban sustraídas" (2012:61). La historia social contemporánea podría calificarse como un proceso de creciente formalización jurídica de las relaciones sociales. Así como Karl Polanyi (1992) indicó que una vez que se desarrolla el mecanismo del mercado se subvierten todas las relaciones existentes (la gran transformación que da título a su célebre obra), la "juridificación" implica un proceso de regulación jurídica de las relaciones sociales, de codificación y formalización de las expectativas recíprocas de quienes se ven involucrados en ellas, a la vez que de las garantías implícitas y explícitas que se establecen al contar con una ultima ratio para recomponer el equilibro roto ante un eventual incumplimiento.

Por lo tanto, la novedad es una nueva ola de juridificación de cuestiones que se encontraban reguladas de manera autónoma o informal (O’Donnell, 2008b), sostenida sobre todo en la expansión global o en palabras de Danilo Zolo (2005) "el espacio jurídico

\footnotetext{
${ }^{3}$ El artículo sintetiza reflexiones en el marco de una indagación teórica y revisión bibliográfica y de casos empíricos que tuvo lugar en el marco del proyecto PICTO financiando por la Agencia Nacional de Promoción Científica y Tecnológica (Argentina), denominado "Acceso a la justicia y marginaciones sociales. Líneas estratégicas provenientes del activismo judicial y social en la región metropolitana de Buenos Aires".
} 
global"4mediante el convencionalismo y la diplomacia de cumbres con la consecuente penetración del campo jurídico (Bourdieu, 2000) en ámbitos sociales diversos, "publificando" asuntos privados, y redefiniendo e invadiendo relaciones domésticas e íntimas.

\section{El protagonismo de los derechos en la escena pública}

La"justiciabilidad" de los derechos sociales se presenta como la posibilidad que un juez ordene una reparación, tal como ocurre con la violación de un derecho civil o político, o bien de reclamar el cumplimiento de las obligaciones que constituyen el objeto del derecho (Artigas, 2005). Distintos factores se combinan para explicar el incremento de los reclamos judiciales vinculados con la satisfacción de derechos sociales en la región en el nuevo siglo: el proceso de democratización y la consecuente restauración del estado de derecho; el deterioro de las condiciones económicas y sociales como consecuencia del proceso denominado de "ajuste estructural", que el periodo de recuperación posterior no logró resolver; la existencia de bloqueos o "callejones sin salida" en los canales tradicionales de la democracia representativa; la ausencia de respuestas adecuadas de los poderes ejecutivo y legislativo ${ }^{5}$; reformas constitucionales que ampliaron el catálogo de derechos de ciudadanía garantizados por el Estado e incluyeron nuevas herramientas para su protección. Dicha formalización constitucional y legal abrió el camino de disputas y conflictos en el terreno judicial incorporando en un escenario corriente la participación de los tribunales en diversas cuestiones políticas.

En tal escenario, aumentó la búsqueda de patrocinio y asesoramiento legal para el reclamo y la defensa de los derechos sociales, incrementando la demanda de servicios para acceder a la justicia; a la vez, surgieron sentencias innovadoras, de diferentes tribunales, fundamentadas en instrumentos internacionales de derechos humanos. La movilización legal de casos colectivos e individuales patrocinados por organizaciones sociales y defensores oficiales fue ganando espacio

\footnotetext{
${ }^{4}$ Zolo(2005) hace un recorrido por las instituciones supranacionales que se perfilan como sujetos del ordenamiento jurídico internacional. Junto a los tratados, a las convenciones y a las costumbres surgen nuevas fuentes del derecho internacional como los actos normativos de las autoridades regionales, la jurisprudencia de los tribunales penales ad hoc, los veredictos de los tribunales arbitrales. Es sobre este último campo y en torno a las elaboraciones normativas de los grandes estudios jurídicos que trabajan en el derecho comercial, fiscal y financiero el punto que reviste mayor interés de la obra.

5 Si bien no será profundizado en este trabajo, la justiciabilidad de los derechos sociales introduce la tensión entre la legitimidad electoral/mayoritaria y la legitimidad judicial o, en otros términos, el carácter de la justicia como poder contramayoritario y no electivo del Estado y, a la vez, con el tipo de asuntos sobre los cuales el poder judicial está autorizado a participar (Gargarella, 1996).
} 
como estrategia de acción política "por otros medios" y como medio de canalización de demandas frente al Estado.

El "enfoque de derechos"surge como nexo entre la perspectiva de análisis de las políticas públicas y el andamiaje de los derechos humanos. Constituye un marco conceptual para fundar normativamente el proceso de desarrollo humano en principios y estándares internacionales, operacionalmente dirigido a respetarlos, protegerlos y satisfacerlos (CEPAL, 2006; Abramovich y Pautassi, 2006). El enfoque pretende integrar tales principios en la legislación nacional, en especial en los programas, planes y procesos de desarrollo definiendo con mayor precisión no sólo aquello que el Estado no debe hacer sino también aquello que sí debe hacer, a fin de lograr la realización de los derechos civiles y políticos como así también de los derechos económicos, sociales y culturales. Esa nueva concepción supera la histórica tradición de conceptualizarlos como un medio para imponer límites a las formas abusivas del poder del Estado y los convierte en un programa que puede orientar las políticas estatales. El conjunto de principios que se desprenden del sistema internacional de derechos humanos constituye parámetros relativamente objetivables en tanto analizadores de las políticas existentes permitiendo demostrar la brecha con los estándares del enfoque. Entre los más relevantes se encuentran: contenido mínimo de los derechos, máximo de los recursos disponibles, prohibición de regresividad, igualdad y no discriminación, especial protección a los grupos sociales más vulnerables, participación de los receptores/destinatarios de las políticas, producción y acceso a la información pública y acceso a la justicia (Pautassi y Royo, 2012). En síntesis, sus principios se constituyeron también en un potenciador de los reclamos judiciales. América Latina estuvo a la cabeza de la actuación de los tribunales en casos sobre derecho sociales (Abramovich y Pautassi, 2009; CELS, 2008; Arcidiáconoet al, 2010; Siederet al, 2008)6.

El discurso delos derechos atravesó el campo de las disputas políticas.Invocados en manifestaciones públicas por políticos y funcionarios, organizaciones de la sociedad civil, organismos internacionales y de asistencia crediticia, asuntos que se expresaban con otras denominaciones y designaciones se enunciaron mediante ese código. En materia de programas sociales, en muchas ocasiones se trató de unaresignificación y actualización política de los discursos sobre "viejos problemas" que pasaron a ser presentados en "clave de derechos" (Smulovitz, 2008) apropiándose de un repertorio para legitimar políticas; en lo sustantivo, no implicaron una adecuación a una visión que a priori pudiera ser considerada próxima a derecho, sino solamente evidenciaron la intencionalidad de hacerlo funcional,

${ }^{6}$ La Red DESC ofrece una base de datos de jurisprudencia a nivel mundial. Allí puede consultarse los más variados casos. Para mayor información cfr. https://www.escrnet.org/es/jurisprudencia 
capitalizando sus "virtudes estratégicas" en el plano discursivo y adaptándose a la "corrección política" de la tendencia internacional7.

Una aclaración necesaria. En una distinción clásica, Esping Andersen (2000) diferenció tres principios "típico ideales" de producción y distribución de bienestar por parte del Estado que pueden ser entendidos como tres tipos de convenios de derechos sociales: el residual, que limita su ayuda a unos estratos de riesgo específico dividiendo a la sociedad entre una mayoría de ciudadanos autosuficientes que pueden asegurarse por medios privados (de mercado o sin fines de lucro) y una clientela dependiente del Estado; el universalista considera que los derechos sociales son incondicionales y que los riesgos se deben compartir bajo una misma cobertura; por último, el corporativista concibe que los riesgos se comparten en función de la pertenencia a un estatus (generalmente el grupo profesional) expresando niveles de diferenciación entre grupos y ramas de actividad de la economía: los derechos sociales suelen asociarse a las condiciones de participación en el mercado formal de trabajo y la idea de la "contribución" mediante el aporte compulsivo de patrones y empleados.

Pese a los avances en materia de reconocimiento de los derechos sociales y a la incorporación del discurso de "derechos", en la región sigue persistiendo un esquema de provisión de bienestar caracterizado por una oferta de beneficios asistencialesdestinados a diferentes grupos definidos como vulnerables, soluciones basadas en el supuesto de la "responsabilización individual" por parte de los receptores que es usual en los formatos más focalizados y residuales social, falta de participación de los protagonistas/receptores en la construcción de la política, débiles mecanismos de control y accountability y falencias en materia de información pública (tanto en términos de producción como de acceso) (Pautassi, 2010; AUTOR, 2012).

Los procesos de "movilización legal" fueron dirigidosdesde arriba mediante la acción de los actores de la élite, entre quienes se cuentan los tribunales y especialmente las cortes constitucionales; desde abajo, mediante la movilización de diversos grupos sociales; e incluso desde el

\footnotetext{
7 El caso del Programa Jefas y Jefes de Hogar Desocupados (PJJHD) en Argentina representa un ejemplo de ese proceder. El programa surgido en 2002 fue denominado "Derecho Familiar de Inclusión Social" rompiendo discursivamente con las denominaciones previas de los programas similares. Sin embargo, aproximadamente unos 200 casos tuvieron por objeto impugnar la imposición sin fundamentos y en los hechos de una fecha de cierre para la postulación (repentinamente planteada por las autoridades a pocos meses de su surgimiento). Se dictaron numerosas sentencias, todas similares, sin emitir un mensaje contundente al Poder Ejecutivo tendiente a evitar la repetición de casos similares. Pese a acatar las sentencias, el gobierno omitió modificar la política, ni siquiera intentó modificar su estrategia de defensa judicial de modo de evitar continuar siendo condenado (Cfr. Arcidiácono, Fairstein y Kletzel, 2009).
} 
extranjero, mediante distintas formas de activismo en tribunales internacionales o de terceros países (Siederet. al, 2008).El litigio debe ser interpretado como una forma de participación en el juego político, un uso de la "voz" (en el sentido de Albert Hirschman, 1977) que intenta, a través de la acción sobre y desde uno de los poderes del Estado, incorporar demandas sociales en la agenda pública por otras vías. La oposición que suele establecerse entre la movilización política y la movilización legal, planteándose como un dilema entre la autonomía de la acción colectiva de los movimientos sociales y el paternalismo emergente de la vía judicial, pierde sustancia. A la luz de la experiencia es razonable pensar a la movilización legal como una pieza del repertorio de la movilización política mediante los estandarizados procedimientos del reclamo jurídico. Dicha estrategia puso en el centro de la escena el problema de la "afiliación" de los sujetos (Zolo, 1997). El acceso a las redes de incidencia legalno puede soslayarse pues es habitual que los sectores más desaventajados presenten menor acceso a la justicia debido a la falta de vínculos con redes de incidencia legal, capaces de patrocinar acciones judiciales. A la vez, los actores vinculados con estas redes están atravesados por agendas propias, las cuales actúan como filtros que condicionan y seleccionan la incorporación de un asunto o problema en calidad potencial de "casojudicial".

Algunos autores presentan una posición crítica respecto de la justiciabilidad de los derechos sociales. Pierre Rosanvallon (1995) resalta el peligro de la radicalización de un individualismo que promueva una intervención estatal compensadora y reparadora de la situación del individuo perdiendo el carácter de reconstrucción del vínculo social en el cual se inscribe el contrato de protección propio del Estado de Bienestar.

En la región son comunes los litigios orientados tanto a reclamos individuales como colectivos; demandas por prestaciones que fueron interrumpidas o que excluyeron a un grupo; otras que aspiraron a la generación de nuevas prestaciones; demandas que implicaron erogaciones estatales y otras que no; presentaciones legales rechazadas por problemas de legitimación de los patrocinantes; sentencias favorables con diversos niveles de cumplimiento; litigios que abrieron canales de diálogo con otros actores, incluyendo otros poderes del Estado, y otros que generaron mayor nivel de tensión; casos individuales que resultaron beneficiosos para los peticionantes pero que no implicaron transformaciones de mayor alcance en la política pública.

Como cualquier otra acción social, los litigios acarrearon consecuencias no deseadas. En algunos casos, en lugar de influir virtuosamente en las decisiones de diseño promovieron la adopción de actitudes conservadoras por parte de los hacedores de la política al desconocer, 
incluso discursivamente, el enfoque de derechos estableciendo soluciones programáticas que evitaron las posibilidades de otorgar poderes a sus receptores. En otros casos, el acceso a la justicia generó nuevos mecanismos de segregación social exacerbando los conflictos al interior de una población en igual condición de riesgo no cubierta por la demanda judicial y aumentando el malestar de quienes no tuvieron acceso a las redes de patrocinio legal; se evidenciaron también falencias del poder judicial para abordar conflictos de carácter colectivo, que fueron procesados como conflictos privados entre partes en igualdad de condiciones.

Las consecuencias del proceso de justiciabilidadno se concentran únicamente en el potencial punitivo de las decisiones judiciales (Smulovitz, 2005); tienen, además, efectos simbólicos al reconocer los problemas y sus sujetos como víctimas de las violaciones de derechos (Mc Cann, 1991 y Galanter, 1983), en el marco de una retórica de derechos que domina en forma creciente la escena pública (Martin, 2012). Además de la satisfacción parcial de las demandas, la intervención de los jueces puede otorgar legitimidad y reconocimiento público a los demandantes, transformando sus peticiones en reclamos legales y en promesas oficiales incumplidas, y a los jueces en "guardianes de promesas anteriores" (Garapon, 1997: p20).

En apretada síntesis, la justiciabilidad se presenta como una estrategia política abierta, compleja, ambivalente y controvertida, en pleno desarrollo, enmarcada en una transformación de la esfera pública donde el discurso de los derechos fue adquiriendo un papel protagónico. A la vez, el reconocimiento de esos derechos (en el mejor de los casos) nos conecta con el clásico problema del diseño e implementación de las políticas sociales y de los satisfactores de bienestar en sociedades de mercado, que complejiza la potencialidad de los fenómenos descriptos representando un elemento central a la hora de comprender la disputa política por la provisión de bienestar, que recientemente se traslada al campo de los tribunales.

\section{Políticas sociales, derechos sociales, satisfactores y mercado}

El alcance de los derechos sociales se vincula con la intensidad y la dinámica de la disputa política y la lucha social, en dos órdenes: en términos de las necesidades cubiertas, esto es, del tipo de riesgos sociales a los cuales se ve expuesta la población (vejez, enfermedad, desempleo, etc.) y respecto de los sujetos de esos derechos (pobres e indigentes, trabajadores, minorías, etc.).

Las políticas sociales se vinculan con las formas históricas mediante las cuales los Estados protegieron legalmente la satisfacción de necesidades sociales (que pueden ser razonablemente llamadas como 
derechos sociales). EspingAndersen (1993:60) señala: "En la historia de la politica social, los conflictos han girado principalmente en torno a qué grado de inmunidad de mercado sería permisible; es decir, los recursos, la extensión y la calidad de los derechos sociales". Desde el campo de la economía política del bienestar, el concepto de "desmercantilización" se convierte en un elemento clave para vincular politicas sociales y derechos. Esping Andersen (2000), sobre nociones desarrolladas por Karl Polanyi ([1944]1992) y Claus Offe (1990), indica que tal designaciónaspira a captar el grado en que los estados debilitan el nexo monetario al garantizar unos derechos independientes de la participación en el mercado. Es decir, se trata de la extracción de una relación social del circuito mercantil y su adscripción a la regulación estatal ${ }^{8}$.

Ahora bien: ¿por qué ciertas necesidades son definidas, tratadas, transformadas o convertidas en derechos? Stephen Holmes y CassSunstein (2011: p.34) definen pragmáticamente a los "derechos" como "intereses importantes que individuos o grupos pueden confiablemente proteger con los instrumentos del gobierno" entendiendo a los derechos legales como aquellos que tienenel respaldo presupuestario del Estado para ponerlos en vigencia.

Evidentemente existen en una sociedad necesidades infinitas, sin embargo, no todas ellas alcanzan ese estatuto. ÁgnesHeller (1996) entiende a los derechos como "permisos": en la medida en que las necesidades son atribuidas y legalmente codificadas, pueden ser reclamadas. La característica de la sociedad capitalista es que los satisfactores de esas necesidades se encuentran cuantificados, "monetizados", y constituye la paradoja del efecto "liberador" del mercado: mientras elimina la adscripción a cualquier otra determinación acentúa el problema de la distribución. En ese punto, bajo el supuesto de que todos los satisfactores pueden ser comprados y vendidos, la garantía de acceso a ese satisfactor se reduce a asignar un poder de consumo a quien no puede asumir el coste por sí mismo, es decir, a reconocer ese satisfactor como un derecho social. En otras palabras, a "desmercantilizar" el acceso a ese satisfactor.

La historia de la política social en los últimos dos siglos ilustra respecto del incremento decisivo de la participación estatal en la producción de satisfactores expresando el creciente proceso de desmercantilización de la posición de las familias. La presencia de los mercados es el hecho distintivo de las sociedades contemporáneas. Los satisfactores protegidos como derechos sociales suponen entonces un tipo de relación de los individuos y los grupos sociales con el mercado,

\footnotetext{
8 En rigor, a partir de la crítica feminista se incorporó la esfera de la familia como fuente protagónica de la producciónde bienestar (cfr. Esping Andersen, 2000) Asimismo autores como Adelantado et. al (1998) agregan a la esfera relacional (organizaciones sociales y comunidad) como una cuarta fuente de producción de bienestar.
} 
específicamente una relación de inmunidad y protección, dado que la tensión entre la capacidad de satisfacer ciertas necesidades básicas adscriptasy el nivel de participación de cada individuo en el mercado constituye la principal fuente de incertidumbre y riesgo social. El punto es central: siguiendo a Polanyi (1992), una economía de mercado es un sistema económico regido, regulado y orientado por los mercados: el orden de la producción y distribución es confiado a ese mecanismo autorregulador y se espera que los individuos obtengan sus ingresos y rentas debido a su participación en los mercados. En otras palabras, cuando Heller define a las necesidades como cuantificables y monetizadas, es decir, asumiendo que esos satisfactores son mercancías potencialmente disponibles para todos, el problema se concentra en la capacidad adquisitiva de cada individuo para satisfacer sus necesidades en el mercado.

En el contexto de la presente discusión, ello tiene al menos dos implicancias: por un lado, es necesario que todos los satisfactores se encuentren disponibles en el mercado, es decir, que puedan ser comprados y vendidos. Sin embargo, la evidencia histórica contradiceesa afirmación provisoria y obliga a una intervención sistémica de carácter político para asegurar su producción en cantidad y calidad, de modo tal de asegurar la reproducción de la "mercancía ficticia" llamada trabajo (Polanyi, 1992). Por otro lado, dada la característica de esa "mercancía ficticia", es imprescindible establecer áreas de producción de satisfactores extrañas al mercado, que suministren esos "bienes de uso" de modo tal que moderen y reduzcan sus condiciones de explotación que, de lo contrario, llevarían a la desaparición física de sus soportes (los individuos). De eso se ocupan los derechos sociales, de atribuir a los individuos y grupos sociales esa garantía, a través de la distorsión del mercado, es decir, de la incorporación de una lógica extra mercantil para asegurar el acceso a esos satisfactores permitiendo su reproducción agregada y continua (estrictamente de la relación social salarial).

La mercantilización del trabajo trajo aparejado un proceso de desmercantilización cuyo modelado fue (y es) obra de los derechos sociales y a la vez es condición necesaria para la continuación de ese proceso de mercantilización. La definición entre la esfera de responsabilidad de cada individuo y la responsabilidad colectiva, entre lo que es una mercancía y entre lo que corresponde proteger como un derecho estructura las nociones vigentes respecto de la justicia social, es uno de los ejes principales de la lucha política y social y encarna el nivel de autonomía que los individuos y grupos sociales logran establecer respecto de la dependencia del mercado.

En apretada síntesis, la garantía de los derechos sociales pone de manifiesto el problema de la producción de los satisfactores, tanto de su costo económico -una restricción- como de la creciente complejidad 
burocrático-administrativa. Sin dudas no hay derechos gratuitos dado que,cualquiera sea su nominación, todos le piden respaldo al erario público;pero el peso de la dimensión prestacional de los derechos sociales enfrenta siempre la necesidad de producir un satisfactor material (ya sean bienes y servicios o transferencias).

Esa especificidad de los derechos sociales incorpora el problema de la mercantilización de la fuerza de trabajo para comprender su sentido histórico en las sociedades de mercado y en la producción de los satisfactores y adquiere especial relevancia en contextos de heterogeneidad estructural, es decir de sociedades desigualmente mercantilizadas $y$, en consecuencia, desigualmente desmercantilizadas.Muchos países de América Latina se constituyeron como sociedades capitalistas donde sobreviven con extensión variable formas cuasi capitalistas de producción: la segmentación y dualidad de los mercados de trabajo combina poblaciones plenamente integradas al sistema económico dinámico con sectores económicamente marginales al modelo de acumulación dominante. ${ }^{9}$ Las características estructuralmente heterogéneas de las sociedades latinoamericanas refuerzan la tensión con el ideal normativo establecido por las aspiraciones igualitarias y universalistas de los derechos.

Esta tensión cobra especial relevancia sobre todo en aquellos países de la región donde continúa vigente la fortaleza simbólica e institucional del sistema de seguridad social organizado a partir de la relación salarial formal10. En otras palabras, donde la forma privilegiada mediante la cual se desarrolló el acceso al goce de los derechos sociales - servicios de atención a la salud, transferencias monetarias a las familias y seguros por vejez y discapacidad- estuvo asociada principalmente a las relaciones asalariadas formales en la que habitualmente participaba el varón cabeza de familia. Este derramaba las protecciones hacia su cónyuge e hijos Estuvo apoyado en un modelo de familia regida según el patrón "varón proveedor/mujer cuidadora", en tanto el trabajo

\footnotetext{
${ }^{9}$ Salvia (2012) actualiza el debate histórico y teórico respecto de la "tesis de la heterogeneidad estructural" en las sociedades latinoamericanas.

${ }^{10}$ Esta afirmación pierde fuerza en los países de América Central donde históricamente los mercados laborales han generado empleo insuficiente, precario, y con actores con frágiles identidades laborales Para mayor información sobre las particularidades regionales Fernando Filgueira (1998) presenta una tipología de los regímenes de bienestar en la región. En apretada síntesis, en primer lugar se encuentran Uruguay, Argentina, Chile y Costa Rica, caracterizados por un universalismo estatificado, es decir, por políticas extendidas pero segmentadas según inserción ocupacional (asalariada o no y según ocupaciones). En segundo lugar identifica países excluyentes, con Estados mínimos y casi nula asignación autorizada de recursos. Ejemplos son El Salvador, Guatemala, Honduras, Nicaragua y República Dominicana. En tercer lugar identifica países duales, en los cuales el universalismo estratificado y el Estado mínimo coexiste en los sectores urbanos y rurales, respectivamente donde se encuentran Brasil y México.
} 
doméstico a cargo de las mujeres en el seno del hogar era un componente esencial en la reproducción social (Pautassi, 2004). La figura del trabajador asalariado fue protagonista del aseguramiento estatal en detrimento de otras categorías sociales. Como producto de la dinámica sindical organizada por rama, y de la diferencial capacidad de movilización colectiva y de presión política de los distintos sectores sociales, al interior de esa categoría aparentemente homogénea se consolidaron prestaciones diferenciales respecto de su extensión y calidad.

\section{Reflexiones finales}

La apelación alos derechos como legitimación de las demandas ciudadanas y en el vocabulario de las políticas públicas en la escena pública,y el incremento de la justiciabilidad en materia de derechos sociales constituyen novedades propias de las últimas dos décadas. Por su parte, el "enfoque de derechos" asumió un rol político como guía de las políticas sociales cuyo basamento ético y su potencial instrumental supone un avance en confrontación con otros paradigmas tecnocráticos o economicistas sostenidos especialmente durante la década de los noventa.

Las estrategias de movilización legal de distintos actores sociales,con capacidad desigualmente distribuida entre las poblaciones, buscaron lograr en los tribunales aquello que, por acción u omisión, tendió a ser negado o desatendido por los canales tradicionales de la democracia representativa. Si bien la dinámica institucional generada a partir de las sentencias merece mayores esfuerzos de investigación empírica, los fallos que ofrecen resultados políticamente sostenibles parecen ser aquellos que se propusieron establecer canales de diálogo entre los poderes del Estado, es decir, que buscaron nutrir una agenda más sensible a través de alimentar las vías tradicionales de formación de la política pública. No obstante, es poco razonable esperar del "activismo judicial" una suplantación del ámbito de definición de la política pública en virtud de ciertas limitaciones estructurales de la acción del Poder Judicial: formación profesional, recursos disponibles, especificidad de su campo de actuación, limitación del saber experto, entre otros asuntos. Los jueces pueden convertirse en actores relevantes dentro del proceso de formación de la política pública, con un peso institucional extraordinario en la agenda, en especial cuando promueven y facilitan la interrelación con los otros poderes del Estado. Sin embargo, podrán continuar las sentencias ejemplares, de alcance individual o colectivo, pero difícilmente se adviertan cambios sustanciales en la dinámica y orientación de la política pública únicamente a través de esos medios.

La especificidad conceptual de los derechos sociales requiere de una cercana consideración de los problemas asociados al nivel de 
mercantilización de la fuerza de trabajo en la medida en que el desarrollo de los arreglos institucionales predominantes en numerosos países de la región se orientó a garantizar la vigencia de los derechos sociales que protegieron la relación social asalariada. Sin embargo, como se señaló, unos de los principales rasgos que definen la heterogeneidad estructural de nuestras sociedades es la sobrevivencia de poblaciones plenamente integradas al mercado de trabajo con poblaciones débil e inestablemente incluidas, aspecto que plantea un desacople con los históricos arreglos bienestaristas.

El protagonismo de los derechos en la agenda públicanos devuelve siempre al problemático escenario de la formulación de las políticas estatales de provisión de satisfactores, vale decir, las múltiples formas de establecimiento de las prioridades políticas y presupuestarias. En suma, el reconocimiento de los derechos sociales como mecanismos de protección de satisfactores "desmercantilizados" de las necesidades sociales refiere al problema estructural de las sociedades modernas: de qué modo se debilita la dependencia de los individuos y grupo sociales respecto de los mercados.

\section{Referencias bibliográficas}

Abramovich, V y Pautassi, L (2009). El enfoque de derechos y la institucionalidad de las politicas sociales. En V. Abramovichy L. Pautassi, (Comps). La revisión judicial de las políticas sociales. Estudio de casos.(pp.279-341). Buenos Aires: Del Puerto.

Adelantado, J.; Noguera, J.; Rambla, X. y Saez, L.(1998).Las relaciones entre estructura y políticas sociales: una propuesta teórica. Revista Mexicana de Sociología 3/98, México, Universidad Autónoma de México, Año LX,3, 133-165.

AUTOR (2008)

AUTOR (2012)

Arcidiácono, P., Fairstein, C. y Kletzel, C (2009). El "enfoque de Derechos en políticas sociales y la experiencia de judicialización del Programa Jefes y Jefas de Hogar Desocupados: ¿Por la buena senda?. En V. Abramovich y L Pautassi (comp.), La revisión judicial de las políticas sociales. Estudio de casos (pp.156-187), Buenos Aires: Del Puerto.

Arcidiácono, P., Espejo Yaksic, N. y Rodriguez Garavito, C. (coord.) (2010) Derechos Sociales: justicia, politica y economía en América Latina. Bogotá: Siglo del Hombre Eds.

Artigas, C.(2005). Una mirada a la protección social desde los derechos humanos y otros contextos internacionales. Serie Políticas Sociales $\mathrm{N}^{\circ}$ 110, Santiago de Chile: CEPAL. 
CELS (Centro de Estudios Legales y Sociales). (2008). La lucha por el derecho.Buenos Aires: Siglo Veintiuno.

CEPAL (Comisión Económica para América Latina) (2006): La protección social de cara al futuro: acceso, financiamiento y solidaridad, Santiago de Chile, CEPAL.

Durkheim, E (2012): "El Estado". En El estado y otros ensayos (pp.59-63), Buenos Aires, Eudeba.

Esping Andersen, G. (2000). Fundamentos sociales de las economías postindustriales. Barcelona: Ariel.

Esping Andersen, G. (1993).Los tres mundos del Estado del Bienestar, Valencia: EdicionsAlfons el Magnanim.

Filgueira, F. (1998). "El nuevo modelo de prestaciones sociales en América Latina: Residualismo y Ciudadanía Estratificada.". En Ciudadanía y política social, (pp. 71-116) San José: FLACSO/SSRC.

Galanter, M. (1983).The Radiating Effects of Courts. En K Boyum y L. Mather (eds.). Empirical Theories About Courts.(pp 35-65)New York: Longman.

Garapon, A(1997). Juez y democracia. Una reflexión muy actual. Barcelona: Flor del Viento Ediciones.

Gargarella, R.(1996). John Ely y la función judicial. En La justicia frente al gobierno. (pp.120-145)Barcelona: Ariel.

Gargarella, R. (2006).Theories of Democracy, the Judiciary and Social Rights. EnR. Gargarella; D. Domingo, y T. Roux (eds.): Courts and Social Transformation in New Democracies. An Institutional Voice for the Poor?(pp.45-64) Aldershot/Burlington; Ashgate.

Giddens, A. (2006) "Estados nacionales y violencia". Revista Académica de Relaciones Internacionales, UAM-AEDRI, 5, 55-78.

Heller, A. (1996). Una revisión de la teoría de las necesidades. Barcelona: Paidós.

Hirschman, A (1977). Salida, voz y lealtad. México: Fondo de Cultura Económica.

Holmes, S. y Sunstein, C. (2011).El costo de los derechos. Por qué la libertad depende de los impuestos. Buenos Aires: Siglo Veintiuno.

Martin, L (2012). Las formas del giro judicial. Judicialización de la política en la democracia argentina contemporánea. En: I. Chereskyy R. Annunziata, (comps.).Sin programa, sin promesa. (pp 147-169) Buenos Aires: Prometeo.

McCann, M.(1991). Legal Mobilization and Social Reform Movements: Notes on Theory and its Application. Studies in Law, Politics and Society.

Nosetto, L. (2014). Reflexiones teóricas sobre la judicialización de la 
política argentina. Documentos y Aportes en Administración Pública y Gestión Estatal Facultad de Ciencias Económicas, Universidad Nacional del Litoral, Santa Fé, 23, 93-123.

O’Donnell, G., Guillermo (2008a) "Hacia un Estado de y para la Democracia". EnDemocracia / Estado / Ciudadanía: hacia un Estado para la Democracia, (pp. 25-62).Lima: PNUD.

O`Donnell, G.(2008b). Epílogo. EnR. Sieder, L. Schjolden y A. Angell(Eds), La judicialización de la política en América Latina.(pp.351-359). Bogotá: Universidad Externado de Colombia. .

Offe, C. (1990). Contradicciones en el Estado de bienestar. Alianza: México.

Pautassi, L. (2004). "Beneficios y beneficiarias: análisis del Programa Jefes y Jefas de Hogar Desocupados de Argentina". EnPolíticas de Empleo para Superar la Pobreza, Santiago: Oficina Internacional del Trabajo.

Pautassi, L. (2010).Indicadores en materia de derechos económicos, sociales y culturales. Más allá de la medición. EnAbramovich, Víctor y Pautassi, Laura: La medición de derechos en las políticas sociales. (pp.189). Buenos Aires: Editores del Puerto.

Pautassi, L y Royo, L. (2012). Enfoque de derechos en las políticas de infancia: indicadores para su medición, Santiago de Chile: CEPAL, UNICEF

Polanyi, K. ([1944] 1992): La Gran Transformación. Crítica del liberalismo económico. Madrid: La Piqueta.

Rosanvallon, P. (1995) La nueva cuestión social.Buenos Aires: Manantial.

Salvia, A. (2012). La trampa neoliberal. Un estudio sobre los cambios en la heterogeneidad estructural y la distribución del ingreso en argentina 1990-2003, Buenos Aires: Eudeba.

Sieder, R.,Schjolden, L. y Angell, A (Eds). (2008).Introducción. En La judicialización de la política en América Latina- (pp.11-37). Bogotá: Universidad Externado de Colombia.

Smulovitz, C.(2008). Petición y creación de derechos: la judicialización en Argentina. En R. Sieder,L. Schojolden, y A. Angell(Eds). La judicialización de la política en América Latina. (pp.193-223). Bogotá; Universidad Externado de Colombia.

Weber, M. [1922] (1944).Economía y Sociedad, México: Fondo de Cultura Económica

Zolo, D. (1997).La ciudadanía en una era poscomunista. Ágora. Cuaderno de Estudios Politicos, Buenos Aires, 7, 99-115.

Zolo, D. (2005). "El espacio jurídico global". En Globalización. Un mapa de los problemas,Bilbao: Edición el Mensajero. 